\title{
Thymic large cell neuroendocrine carcinoma: report of a resected case - a case report
}

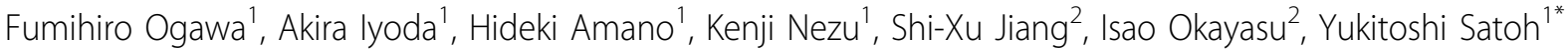

\begin{abstract}
Thymic large cell neuroendocrine carcinomas (LCNECS) are very rare. We here describe a case in which the tumor could be completely resected. A 55-year-old male was admitted to our hospital for treatment of an anterior mediastinal tumor found at a regular health check-up. The patient underwent an extended thymectomy of an invasive thymoma of Masaoka's stage II that had been suspected preoperatively. The tumor was located in the right lobe of the thymus and was completely resected. Final pathological diagnosis of the surgical specimen was thymic LCNEC. The patient underwent adjuvant chemotherapy with irinotecan and cisplatin in accordance with the diagnosis of a lung LCNEC, and is alive without recurrence or metastasis 16 months after surgery.
\end{abstract}

\section{Background}

Primary thymic neuroendocrine carcinomas (NECs) were categorized under the rubric of 'thymomas' until 1972, when Rosai and Higa suggested that these tumors were sufficiently distinctive to warrant classification as carcinoid tumors [1]. Thymic NECs are relatively rare neoplasms that account for only approximately $2 \%$ to $4 \%$ of all anterior mediastinal neoplasms [2]. In 1999, the World Health Organization established thymic epithelial tumor criteria and reclassified thymic carcinoma, referring to NECs as a subtype [3]. In particular, the LCNEC was subclassified in the thymic NECs in accordance with the classification of pulmonary neuroendocrine tumors. Detailed clinical features of thymic LCNECs are still unknown, however, because of their rareness. We described a case with a review of the literature, focusing on the most likely optimal treatment it.

\section{Case presentation}

A 55-year-old Japanese male was admitted to the Kitasato University Hospital for further examination and treatment for an abnormal shadow on the chest $\mathrm{x}$-ray found at a regular health check-up. He had smoked 35 packs per year for 20 years. Chest $\mathrm{x}$-ray films showed a solid mass with a clear border at the right hilum and a negative silhouette sign for the right first arch (Figure

\footnotetext{
* Correspondence: ysatoh@med.kitasato-u.ac.jp

'Department of Thoracic Surgery, Kitasato University School of Medicine, Kanagawa, Japan

Full list of author information is available at the end of the article
}

1). Enhanced chest computed tomography (CT) revealed a solid mass $42 \mathrm{~mm}$ in diameter with a partially unclear margin with the normal thymic tissue in the anterior mediastinum (Figure 2). Magnetic resonance imaging (MRI) using intravenous contrast medium showed isointensity of the mass on both T1- and T2-weighted images (Figure 3,4). Although chest CT and MRI revealed no invasion of the superior vena cava and the innominate vein, the tumor was highly suspected to have invaded the normal thymic tissue. Laboratory findings and results for tumor markers such as CEA (carcinoembryonic antigen), NSE (neuron specific enolase), and ProGRP (pro-gastrin releasing peptide) were all within normal ranges, preoperatively.

Under the diagnosis of invasive thymoma or thymic carcinoid, the patient underwent an extended thymectomy. The tumor was intraoperatively revealed in the right lobe of the thymus without any invasion to the adjacent organs:the aorta, superior vena cava, pericardium, bilateral phrenic nerve, or the right lung. Because the tumor had invaded the right parietal pleura, we also resected the right parietal pleura with a sufficient surgical margin.

Macroscopically, the elastic soft tumor surrounded by thymic fat tissue was $40 \times 35 \times 28 \mathrm{~mm}$ in size. The cut surface was mainly whitish-yellow in color and showed focal necrosis and red bloody spots.

Microscopically, the tumor manifested morphologic features of a carcinoid. The tumor cells were arranged in wide trabeculae with irregular nests separated by thin 


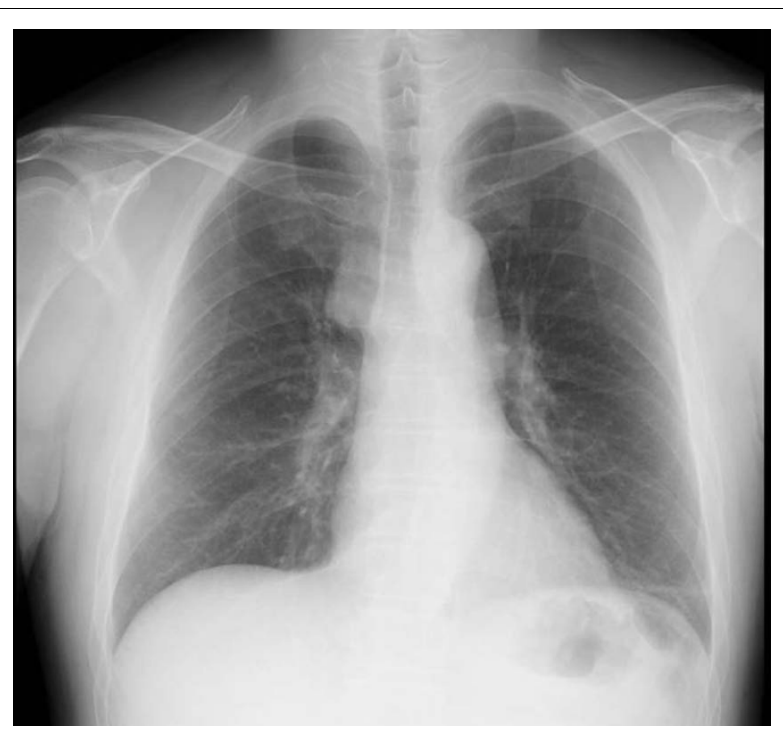

Figure 1 Chest $x$-ray showing a solid mass with a clear border at the right hilum and a negative silhouette sign for the right first arch

fibrovascular stroma, and scattered abortive rosette-like structures (Figure 5). The tumor cells were oval to polygonal in shape with abundant eosinophilic and granular cytoplasm. The nuclear chromatin was granular and the nucleoli were inconspicuous. Small foci of coagulative necrosis were also observed (Figure 6). The average mitotic count was 30 per each of 10 high-power fields (Figure 7), and the Ki-67 indices using MIB-1 immunohistochemical staining ranged from $20 \%$ to $30 \%$. Immunohistochemically, the tumor cells were diffusely positive for chromogranin A (Figure 8), synaptophysin, and neural cell adhesion molecule (NCAM), confirming a neuroendocrine nature. Thus, the final pathological diagnosis of thymic LCNEC was made. The tumor also invaded atrophic normal thymic tissue.

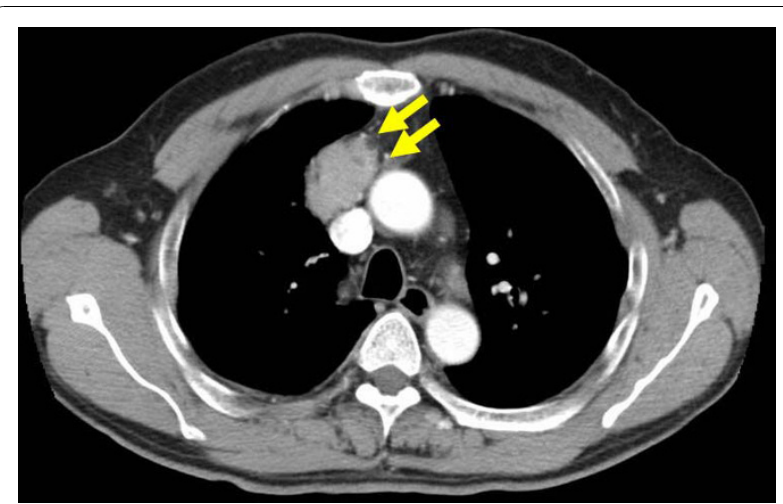

Figure 2 Enhanced chest CT scan revealing a $42-\mathrm{mm}$-sized solid mass with an unclear margin (arrows) with the normal thymus in the anterior mediastinum.

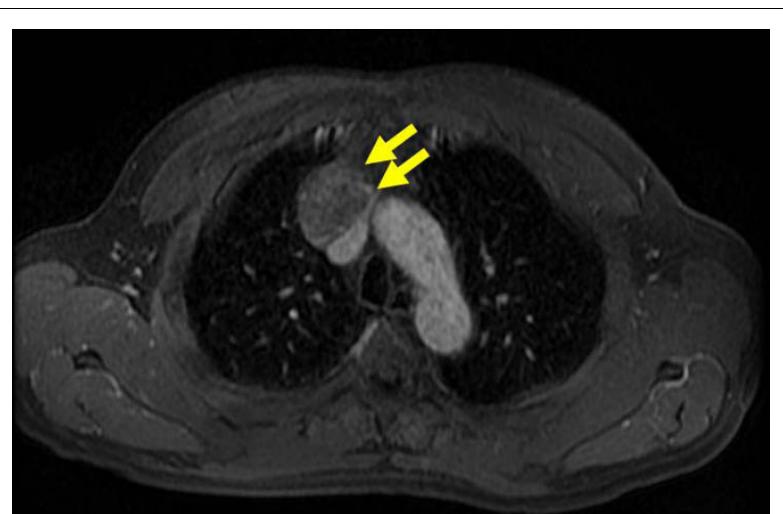

Figure 3 Chest MRI using intravenous contrast medium showed iso-intensity of the mass on a T1-weighted image.

The patient underwent adjuvant chemotherapy based on a platinum doublet containing cisplatin at $60 \mathrm{mg} / \mathrm{m}^{2}$ and irinotecan at $60 \mathrm{mg} / \mathrm{m}^{2}$ for three courses, and is alive without recurrence or metastasis at 16 months after surgery.

\section{Discussion}

The neuroendocrine subtype of thymus tumors is defined on the basis of histopathological features and immunophenotypes. In recent studies [4,5], NECs have been morphologically categorized into four main types: typical carcinoid, atypical carcinoid, LCNEC, and small-cell carcinoma. To our knowledge, LCNECs and small-cell carcinomas are highly malignant and have a poorer prognosis than do other thymic epithelial tumors. The LCNEC is included as a separate entity because of differences from carcinoids in survival rates as well as its incidence and clinical, epidemiologic, histological, and molecular characteristics.

Although chest CT and MRI revealed no invasion to the superior vena cava or the innominate vein in the

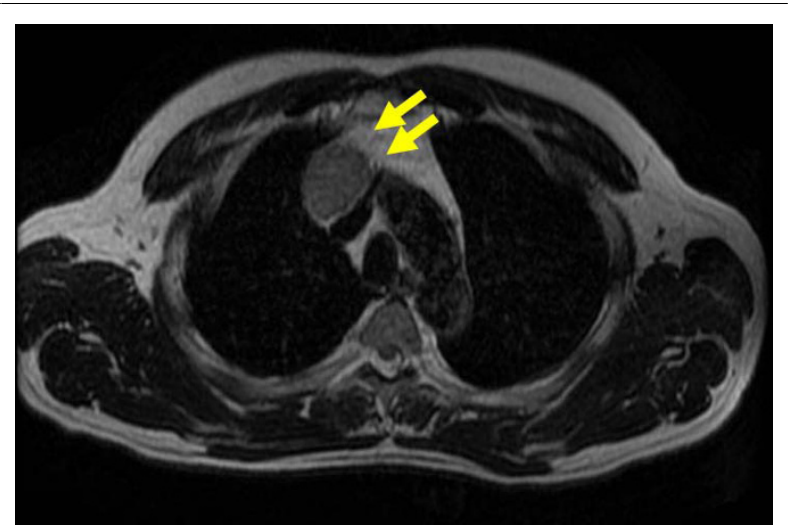

Figure 4 Chest MRI using intravenous contrast medium showed iso-intensity of the mass on a T2-weighted image with an unclear rim (arrows), as with the chest $\mathrm{CT}$, too. 


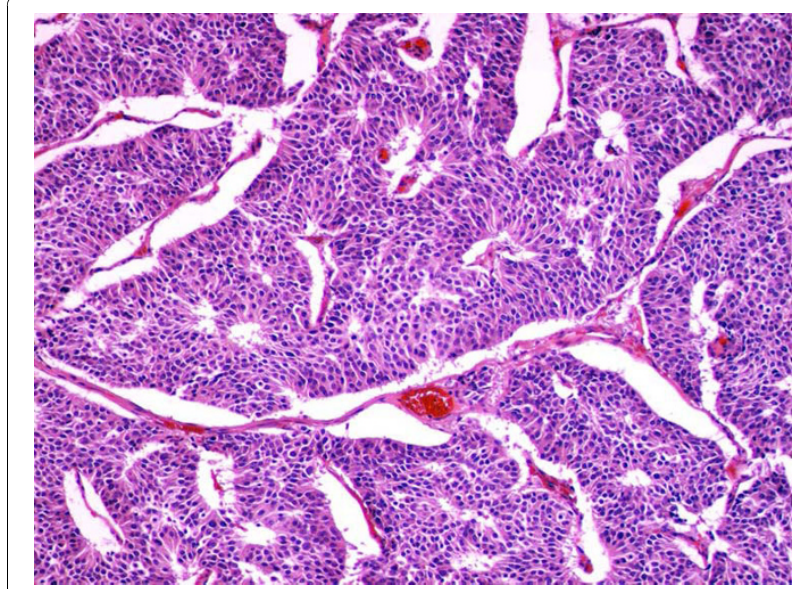

Figure 5 The tumor cells were arranged in wide trabeculae with irregular nests separated by thin fibrovascular stroma, and scattered abortive rosette-like structures were encountered. (hematoxylin and eosin staining, $\times 40$ ).

present case, and T1- and T2-weighted images demonstrated isointensity, the tumor was highly suspected of having invaded the normal thymic tissue due to its unclear rim. Therefore, our preoperative diagnosis was an invasive thymoma or a carcinoid.

For optimal treatment, an accurate pretherapeutic diagnosis is important. However, as a thymic tumor is not always morphologically homogeneous, this may be difficult with a standard needle biopsy. Surgery offers the best chance for a definitive diagnosis and curative treatment of thymic tumors. The differential diagnosis for the anterior mediastinum includes other primary mediastinal tumors, mainly thymoma, paraganglioma, lymphoma, parathyroid adenoma or carcinoma, as well as medullary carcinoma of the thyroid. The most difficult but most important differential diagnosis in this setting is with

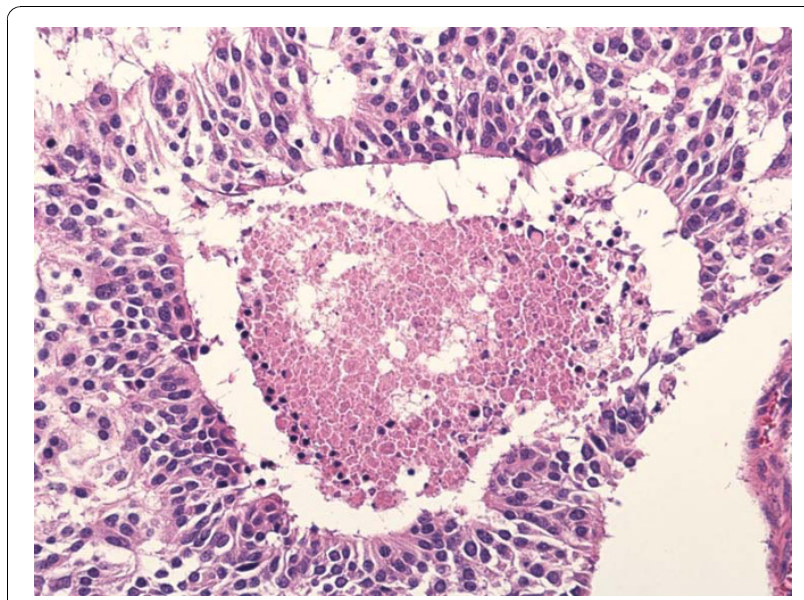

Figure 6 Small foci of coagulative necrosis were also observed. (hematoxylin and eosin staining, $\times 100$ ).

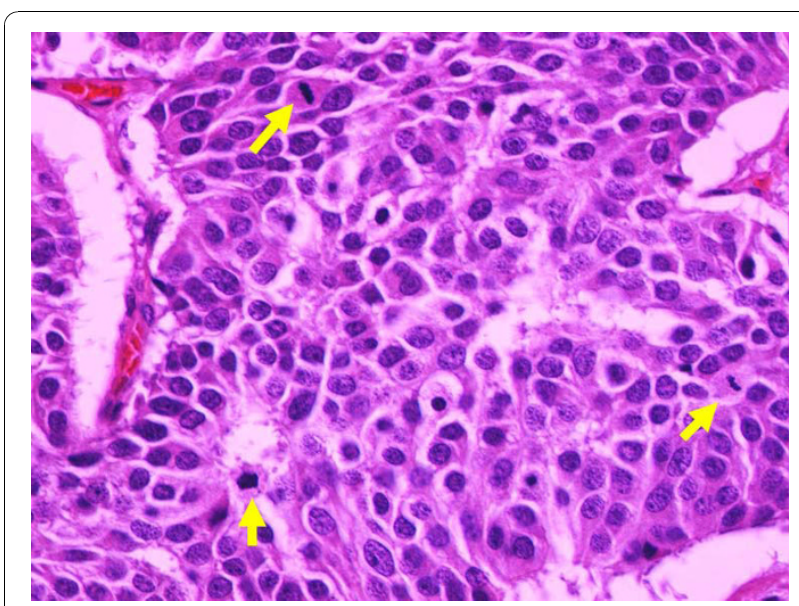

Figure 7 Mitosis(arrows) counts ranged around 30 per 10 highpower fields. (hematoxylin and eosin staining, $\times 400$ ).

thymoma, particularly of the spindle cell type. This latter can often show areas displaying a prominent neuroendocrine appearance with abundant epithelial cells disposed radially around an empty space closely simulating the microacinar growth pattern sometimes observed in carcinoids. To make a successful differential diagnosis, immunohistochemical staining can be helpful. Even though both types of lesions share strong CAM 5.2 positivity, thymomas are negative for neuroendocrine markers (e.g. chromogranin A, synaptophysin, NCAM, and CD56) and may be useful for NECs [6].

Thymic LCNEC is very rare. A search of the PubMed database revealed only a few case reports in the literature [7-11]. Mega et al. reviewed 10 cases of thymic LCNECs in Japan [7]. As seen in Table 1, surgical resection was performed in 8 of the 10 cases, but most of the patients were at an advanced stage of disease and half

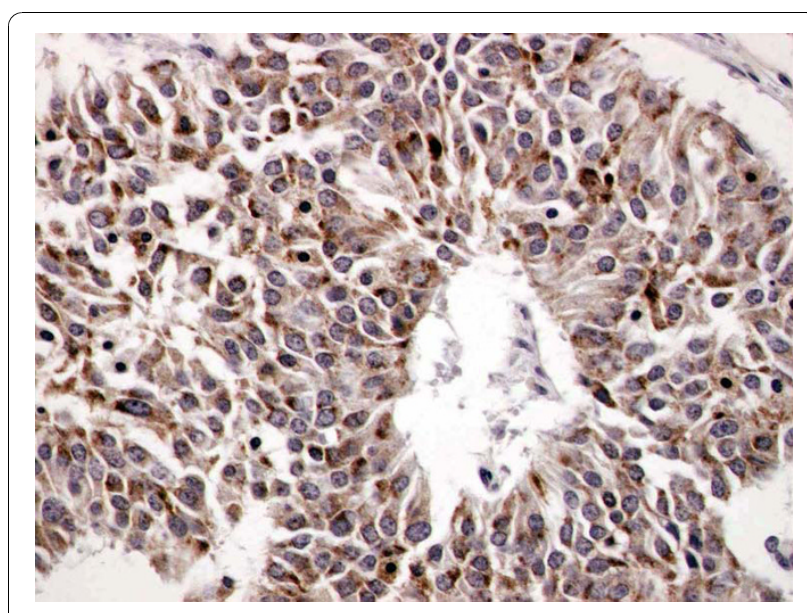

Figure 8 Tumor cells were diffusely positive for chromogranin A $(\times 400)$. 
Table 1 The case report of thymic LCNECs in Japan

\begin{tabular}{|c|c|c|c|c|c|c|c|}
\hline Case & Age & Gender & Report (year) & Size & Masaoka stage & Treatment & Prognosis \\
\hline 1 & 53 & $\mathrm{~F}$ & Kurashima (2002) & 50 & $\mathrm{IVa}$ & $O p+C x+R x$ & N.S. \\
\hline 2 & 50 & M & Miura (2003) & 40 & $\mathrm{IVa}$ & Op & N.S. \\
\hline 3 & 63 & $\mathrm{~F}$ & Hirami (2004) & 50 & $\mathrm{IVb}$ & $O p+R x$ & $4 \mathrm{M}$ Recurrence \\
\hline 4 & 57 & $\mathrm{~F}$ & Nagata (2005) & 70 & $\|$ & $O p+R x$ & $7 \mathrm{M}$ Recurrence \\
\hline 5 & 69 & $\mathrm{~F}$ & Kitagawa (2006) & N.S. & $\mathrm{IVb}$ & $C x$ & N.S. \\
\hline 6 & N.S. & N.S. & Shimokawa (2006) & N.S. & N.S. & N.S. & N.S. \\
\hline 7 & 46 & M & Tao (2006) & N.S. & $\mathrm{IVb}$ & $O p+C x+R x$ & $4 \mathrm{M}$ Recurrence \\
\hline 8 & 63 & $\mathrm{~F}$ & Tao (2006) & N.S. & $\mathrm{IVb}$ & $O p+C x+R x$ & $4 \mathrm{M}$ Recurrence \\
\hline 9 & 67 & $\mathrm{~F}$ & Kou (2007) & N.S. & $\mathrm{IVa}$ & $O p+C x+R x$ & N.S. \\
\hline 10 & 67 & $\mathrm{~F}$ & Mega (2008) & 60 & $\mathrm{IVb}$ & $O p+C x+R x$ & $6 \mathrm{M}$ Recurrence \\
\hline 11 & 55 & M & This Case & 42 & $\|$ & $\mathrm{Op}+\mathrm{Cx}$ & No Recurrence \\
\hline
\end{tabular}

Op: operation Cx: chemotherapy Rx: irradiation N.S.: Not shown

had recurrence. Furthermore, recurrence occurred relatively soon after surgery (range 2 to 7 months) and their prognoses were very poor. Cesar et al. reported [6], the primary mediastinal NEC to represent a separate biologic entity from carcinoids arising at other locations, with disease-free survivals of $50 \%$ at 5 years and $9 \%$ at 10 years for well differentiated tumors (i.e. typical carcinoids), $20 \%$ at 5 years and $0 \%$ at 10 years for moderately differentiated tumors (i.e. atypical carcinoids), and $0 \%$ at 5 years for poorly differentiated tumors. Therefore, it can be considered that a well differentiated grade and complete surgical removal followed by adjuvant therapy offer curative potential and are significant factors for prolonged survival [7-9].

Currently, there is no evidence to support the use of postoperative therapy for Thymic LCNECs. Recent studies [12-14] of LCNEC of the lung recommended postoperative administration of adjuvant chemotherapy with platinum-based combination regimens (e.g. etoposide and others), which is the regimen for small cell lung carcinoma similar to the clinicopathologic and biologic features of LCNEC. Their results showed good prognosis. Platinum-based combination regimens were effective for the patients with LCNEC in their studies. Likewise, we believe that surgery and adjuvant therapy are needed to treat LCNEC in the thymus. Therefore we selected the regimen, cisplatin/irinotecan, for small cell lung carcinoma because Noda et al. revealed that cisplatin/irinotecan provided better results than did cisplatin/ etoposide [15]. And Fujiwara et al. [16] also indicated that irinotecan-based regimens might be as active against LCNEC of the lung as against SCLC. Since recurrence of thymic LCNECs occurs within a short duration after surgery and their prognosis is very poor, we regarded this disease as having an extensive status at resection. Therefore, we selected the regimen, cisplatin/ irinotecan. However, the odalities for adjuvant chemotherapy remain to be defined.

\section{Conclusion}

Because primary thymic LCNECs are very rare, and the patients'prognoses are very poor, along with the lack of experience, a standardized treatment protocol, and the limited literature, all these contributing factors make it a difficult tumor to treat. Additional studies area warranted to determine the optimal treatment of thymic LCNECs.

\section{Consent}

Written informed consent was obtained from patient for publication of this case report and any accompanying images. A copy of the written consent is available for review by the Editor-in-Chief of this journal.

\section{Acknowledgements}

Part of this study was supported by a Grant-in-Aid for Scientific Research (C) from the Japan Society for the Promotion of Science (No.20591676), as well as a grant from the Ministry of Health, Labour and Welfare, Japan (No.19-12).

\section{Author details}

${ }^{1}$ Department of Thoracic Surgery, Kitasato University School of Medicine, Kanagawa, Japan. ${ }^{2}$ Department of Pathology, Kitasato University School of Medicine, Kanagawa, Japan.

\section{Authors' contributions}

FO carried out the manuscript and collected references. YS coordinated all authors. FO and YS underwent this operation, and Al, HA, and KN helped for clinical support with them. SJ and 10 reported pathological findings and took the pathologic pictures. Al and YS helped to draft the manuscript. All authors read and approved the final manuscript.

\section{Competing interests}

The authors declare that they have no competing interests.

Received: 18 August 2010 Accepted: 22 November 2010 Published: 22 November 2010

\section{References}

1. Rosai J, Higa E: Mediastinal endocrine neoplasm of probable thymic origin, related to carcinoid tumor. Clinicopathologic study of 8 cases. Cancer 1972, 29:1061-1074.

2. Wick MR, Rosai J: Neuroendocrine neoplasms of the mediastinum. Semin Diagn Pathol 1991, 8:35-51. 
3. Rosai J: Histological Typing of the Thymus. World Health Organization International Histological Classification of tumours. 2 edition. Berlin: SpringerVerlag; 1999

4. Chetty R, Batitang S, Govender D: Large cell neuroendocrine carcinoma of the thymus. Histopathology 1997, 31:274-276.

5. Moran CA, Suster S: Neuroendocrine carcinomas (carcinoid tumor) of the thymus. A clinicopathologic analysis of 80 cases. Am J Clin Pathol 2000, 114:100-110

6. Cesar AM, Saul S: Neuroendocrine carcinomas (carcinoid tumor) of the thymus. Am J Clin Pathol 2000, 114:100-110.

7. Mega S, Oguri M, Kawasaki R, Hazama K, Iwai K, Kondo S: Large-cell neuroendocrine carcinoma in the thymus. Gen Thorac Cardiovasc Surg 2008, 56:566-569.

8. Tiffet O, Nicholson AG, Ladas G, Sheppard MN, Goldstraw P: A clinicopathologic study of 12 neuroendocrine tumors arising in the thymus. Chest 2003, 124:141-146.

9. Ogawa K, Toita T, Uno T, Fuwa N, Kakinohana Y, Kamata M, Koja K, Kinjo T, Adachi G, Murayama S: Treatment and prognosis of thymic carcinoma: a retrospective analysis of 40 cases. Cancer 2002, 94:3115-3119.

10. Dutta R, Kumar A, Jindal T, Mathur SR: Neuroendocrine carcinoma of the thymus gland with sternal invasion. Interact CardioVasc Surg 2009, 8:694-696.

11. Takezawa K, Okamoto I, Fukuoka J, Tanaka K, Kaneda H, Uejima H, Yoon HE, Imakita M, Fukuoka M, Nakagawa K: Large cell neuroendocrine carcinoma of the mediastinum with alpha-fetoprotein production. J Thorac Oncol 2008, 3:187-189.

12. Iyoda A, Hiroshima K, Moriya Y, Takiguchi Y, Sekine Y, Shibuya K, lizasa T, Kimura H, Nakatani Y, Fujisawa T: Prospective study of adjuvant chemotherapy for pulmonary large cell neuroendocrine carcinoma. Ann Thorac Surg 2006, 82:1802-1807.

13. Rossi G, Cavazza A, Marchioni A, Longo L, Migaldi M, Sartori G, Bigiani N, Schirosi L, Casali C, Morandi U, Facciolongo N, Maiorana A, Bavieri M, Fabbri LM, Brambilla E: Role of chemotherapy and the receptor tyrosine kinases KIT, PDGFR alpha, PDGFRbeta, and Met in large-cell neuroendocrine carcinoma of the lung. J Clin Oncol 2005, 23:8774-8785.

14. Iyoda A, Hiroshima K, Moriya Y, Iwadate Y, Takiguchi Y, Uno T, Nakatani Y, Yoshino I: Postoperative recurrence and the role of adjuvant chemotherapy in patients with pulmonary large-cell neuroendocrine carcinoma. J Thorac Cardiovasc Surg 2009, 138:446-453.

15. Noda K, Nishiwaki Y, Kawahara M, Negoro S, Sugiura T, Yokoyama A, Fukuoka M, Mori K, Watanabe K, Tamura T, Yamamoto S, Saijo N: Japan Clinical Oncology Group: Irinotecan plus cisplatin compared with etoposide plus cisplatin for extensive small-cell lung cancer. $N$ Engl J Med 2002, 346(2):85-91.

16. Fujiwara $Y$, Sekine I, Tsuta $K$, Ohe $Y$, Kunitoh $H$, Yamamoto N, Nokihara H, Yamada K, Tamura T: Effect of platinum combined with irinotecan or paclitaxel against large cell neuroendocrine carcinoma of the lung. Jpn J Clin Oncol 2007, 37(7):482-486.

doi:10.1186/1749-8090-5-115

Cite this article as: Ogawa et al.: Thymic large cell neuroendocrine carcinoma: report of a resected case - a case report. Journal of Cardiothoracic Surgery 2010 5:115.

\section{Submit your next manuscript to BioMed Central and take full advantage of:}

- Convenient online submission

- Thorough peer review

- No space constraints or color figure charges

- Immediate publication on acceptance

- Inclusion in PubMed, CAS, Scopus and Google Scholar

- Research which is freely available for redistribution

Submit your manuscript at www.biomedcentral.com/submit
Ciomed Central 